\title{
Dishevelling Wnt and Hippo
}

\author{
Nam Hee Kim ${ }^{1}$, Yoonmi Lee ${ }^{1}$ E Jong In Yook ${ }^{1,2, *}$ \\ ${ }^{1}$ Oral Cancer Research Institute and ${ }^{2}$ Department of Oral Pathology, Yonsei University College of Dentistry, Seoul 03722, Korea
}

As highly conserved signaling cascades of multicellular organisms, Wnt and Hippo pathways control a wide range of cellular activities, including cell adhesion, fate determination, cell cycle, motility, polarity, and metabolism. Dysregulation of those pathways are implicated in many human diseases, including cancer. Similarly to $\beta$-catenin in the Wnt pathway, the YAP transcription co-activator is a major player in Hippo. Although the intracellular dynamics of YAP are well-known to largely depend on phosphorylation by LATS and AMPK kinases, the molecular effector of YAP cytosolic translocation remains unidentified. Recently, we reported that the Dishevelled (DVL), a key scaffolding protein between canonical and non-canonical Wnt pathway, is responsible for nuclear export of phosphorylated YAP. The DVL is also required for YAP intracellular trafficking induced by E-cadherin, $\alpha$-catenin, or metabolic stress. Note that the p53/LATS2 and LKB1/AMPK tumor suppressor axes, commonly inactivated in human cancer, govern the reciprocal inhibition between DVL and YAP. Conversely, loss of the tumor suppressor allows co-activation of YAP and Wnt independent of epithelial polarity or contact inhibition in human cancer. These observations provide novel mechanistic insight into (1) a tight molecular connection merging the Wnt and Hippo pathways, and (2) the importance of tumor suppressor contexts with respect to controlled proliferation and epithelial polarity regulated by cell adhesion. [BMB Reports: Perspective 2018; 51(9): 425-426]

*Corresponding author. E-mail: jiyook@yuhs.ac

https://doi.org/10.5483/BMBRep.2018.51.9.179

\section{Received 1 August 2018}

Keywords: Dishevelled, Hippo pathway, Tumor suppressor, Wnt signaling, YAP

Abbreviations: AMPK, AMP-activated kinase; APC, Adenomatous polyposis coli; EMT, Epthelial-mesenchymal transition; LATS, Large tumor suppressor; LKB1, Liver kinase B1; YAP, Yes-associated protein

Perspective to: Yoon Mi et al., (2018) Nature Communications. 9:301, DOI: 10.1038/s41467-018-04757-w
The Human Genome Project revealed a remarkably small number of ligand-mediated signaling pathways, such as the Wnt and TGF- $\beta$ superfamily, involved in a biologically diverse range of human diseases. Thus, molecular components within a limited number of pathways are dynamically interacting into higher order signaling networks that dictate the biological outcomes of pathway activity. Typically, post-translational modification, molecular interaction, and intracellular trafficking of signaling molecules account for diverse biological aspects of a pathway.

The Hippo signaling has emerged as a critical regulator of organ size, tissue homeostasis, and patterning underlying cell-to-cell contact inhibition, loss of Hippo leading to organ overgrowth and cancer proliferation. The intracellular core of the Hippo pathway consists of a kinase cascade regulating nuclear-cytoplasmic shuttling of YAP (Moroishi T et al (2015) Nat Rev Cancer 15, 73-79). The Lats1 or Lats2 are well-known kinases resulting in cytoplasmic translocation of YAP. In the normal physiologic context of epithelial cells, adherens and tight junctions mediate contact inhibition of cell growth in a Lats kinases-dependent manner. Interestingly, metabolic starvation relocates nuclear YAP into cytoplasm via AMPK (Mo JS et al (2015) Nat Cell Biol 17, 500-510). While the phosphorylation-dependent YAP shuttling constitutes a core of the Hippo pathway, the molecular effector by which YAP translocates has until now been unknown.

Hyperactivation of the Wnt pathway constitutes a key feature in human cancer, including colorectal cancer, which has frequent APC or $\beta$-catenin mutation. The importance of the Wnt pathway is also highlighted by its coordinate control of the transcriptional program underlying Snail-mediated EMT. In canonical Wnt signaling, well-known scaffolding proteins such as APC, Axin and DVL function as critical intracellular regulators of $\beta$-catenin dynamics. While the Axin is regulated by Wnt co-receptor LRP5/6, the DVL acts directly downstream of Frizzled receptors. As an essential component of the Wnt pathway, the enigmatic DVL transduces signals into three separate signaling branches, the canonical ( $\beta$-catenin dependent), non-canonical ( $\beta$-catenin independent), and $\mathrm{Wnt}_{-\mathrm{Ca}}{ }^{++}$ pathways. DVL interacts with a wide range of partner proteins, revealing its diverse functions in areas including cellular differentiation, inflammation, neurodegeneration, cell polarity and social behavior. DVL consists of three homolog genes in mammal, DVL1, DVL2, and DVL3. These multifunctional proteins mediate complex signal transduction via conserved

ISSN: 1976-670X (electronic edition)

Copyright (C) 2018 by the The Korean Society for Biochemistry and Molecular Biology

(c) This is an open-access article distributed under the terms of the Creative Commons Attribution Non-Commercial License (http://creativecommons.org/licenses/by-nc/4.0) which permits unrestricted non-commercial use, distribution, and reproduction in any medium, provided the original work is properly cited. 




Diagram 1. Schematic model for phosphorylation-dependent YAP translocation by DVL. When the Hippo pathway is activated, Lats1/2-phosphorylated YAP leads to inhibition of the nuclear transcriptional activity through cytoplasmic translocation. The phosphorylated YAP is exported by DVL into cytoplasmic space. Note that p53 and LKB1/AMPK tumor suppressors maintain Lats1/2 activities which are responsible for YAP trafficking controlled by the contact inhibition from tight/adherens junctions or metabolic regulation.

domains: the DIX and PDZ domains serve for the canonical Wnt pathway while the PDZ and DEP domains serve for non-canonical Wnt. Although DVL2 has been studied in developmental contexts due to its ubiquitous abundance in various tissues, we found that the DVL3 isoform is mainly expressed in human cancer cells in terms of transcript in clinical samples and protein abundance in cancer cell line panels.

Given the importance of contact inhibition in multicellular organisms, the reciprocal regulation between Wnt and Hippo is of central interest in development as well as in human cancer. While large bodies of recent studies have focused on the role of YAP in Wnt suppression via DVL, a ligand-specific receptor of Hippo has not yet been found. We therefore raised the hypothesis that DVL harbors a function which specifically enables YAP to tightly connect Hippo and Wnt. Indeed, DVL scaffolding protein drives the nuclear export of phosphorylated YAP. The DVL contains conserved nuclear export signals (NES, M/LxxLxL) located next to the DEP domain, the NES being responsible for YAP cytoplasmic translocation. Importantly, the DVL is required for YAP cytoplasmic translocation induced by tight contact inhibition, E-cahderin, $\alpha$-catenin or AMPK activation, indicating that Wnt scaffolding protein DVL is an important shuttling effector in the Hippo pathway.

The tumor suppressors p53 and LKB1 are most frequently inactivated in human cancer. The Lats2 is one of the transcriptional targets of p53, and AMPK is a well-known downstream cascade of LKB1. Thus, the loss of p53 and LKB1 in cancer abolishes Lats2 and AMPK activities essentially required for DVL's role in YAP trafficking. Note that p53 transcription activity also suppresses canonical Wnt and the Snail-mediated EMT program (Kim NH et al (2011) Sci Signal 4 , ra71). Therefore, the intact tumor suppressor governs the reciprocal regulation between Wnt and Hippo, resulting in balanced cell migration and controlled proliferation by contact inhibition. In turn, loss of tumor suppressor in human cancer abolishes DVL's role in YAP translocation and allows consistent activation of Wnt and nuclear YAP via feed-forward activation. Note that Wnt ligands activate YAP via the G $\alpha /$ Rho/Lats $1 / 2$ cascade (Park HW et al (2015) Cell 162, 780-794). Given the YAP phosphorylation-dependent interaction with DVL, Wnt ligands (such as Wnt1 or Wnt 3a) dissociate DVL-YAP interaction, resulting in co-activation of canonical Wnt and nuclear YAP activity. Therefore, intact p53 or LKB1 tumor suppressor function maintains epithelial polarity, restricting co-activation of canonical Wnt and nuclear YAP activities (Diagram 1). Conversely, hyperactivation of Wnt coupled with loss of tumor suppressor constitutes a core signaling network, allowing the uncontrolled proliferation and metastatic potential of human cancer.

These observations provide several insights into highly conserved signaling pathways coupled with tumor suppressor contexts. First, although the Wnt and Hippo pathways are largely regarded as independent, they may be closely linked via DVL. Second, the Wnt and Hippo comprise a central core signaling, balancing cell growth by contact inhibition and migratory potential, such as in wound healing under physiologic condition. Lastly, loss of tumor suppressor function disrupts DVL's role in the Hippo pathway allowing the feed-forward co-activation of Wnt and YAP commonly observed in human cancer.

In conclusion, this study provides mechanistic insight into how the Wnt and Hippo pathways coordinate with each other during development and human cancer. Specifically, the Wnt scaffolding protein DVL functions as a molecular effector of YAP nuclear-cytoplasmic shuttling, being regulated by Hippo pathway and metabolic cues. Further study is thus needed to identify the role of DVL in epithelial polarity, adhesion and metabolism.

\section{ACKNOWLEDGEMENTS}

We thank E. Tunkle for preparation of the manuscript. This work was supported by grants from the National Research Foundation of Korea (NFR-2017R1A2B3002241, NRF-2016R 1E1A1A01942724, NRF-2014R1A6A3A04055110) funded by the Korean government (MSIP) and a grant from the Korea Health Industry Development Institute (KHIDI) funded by the Ministry for Health \& Welfare Korea (HI17C2586). 